\title{
The stochastic piston problem
}

\author{
G. Lin, C. H. Su, and G. E. Karniadakis* \\ Division of Applied Mathematics, Brown University, 182 George Street, Providence, RI 02912
}

Edited by Richard V. Kadison, University of Pennsylvania, Philadelphia, PA, and approved September 30, 2004 (received for review August 11, 2004)

We obtain analytical solutions for the perturbed shock paths induced by time-varying random motions of a piston moving inside an adiabatic tube of constant area. The variance of the shock location grows quadratically with time for early times and switches to linear growth for longer times. The analytical results are confirmed by stochastic numerical simulations, and deviations for large random piston motions are established.

$\mathbf{T}$ he English physicist James Joule was perhaps the first to use the concept of a moving piston in order to demonstrate the mechanical equivalent of heat in his pioneering studies, almost 2 centuries ago. The moving piston has also been used extensively in fundamental studies of fluid mechanics and shock discontinuities in the last century, and this now classical problem has been solved analytically in one dimension and also in higher space dimensions $(1,2)$. It is well known that a shock wave propagating into a stationary fluid sets it into motion and raises its pressure, temperature, and density. This situation can be physically realized by a planar, cylindrical, or spherical piston moving at specified speed into a stagnant fluid. In gas dynamics, in particular, in the context of normal shock waves, the one-dimensional classical problem describes a piston moving at constant speed in a tube of constant area and adiabatic walls; the shock wave is created ahead of the piston. Closed-form analytical solutions of this flow problem with general time-dependent piston speeds are difficult to obtain; see semianalytical solutions in ref. 3 for accelerating and decelerating pistons that are valid only for short times.

In this article, we revisit the one-dimensional piston problem within the stochastic framework; i.e., we allow for random piston motions that may be changing in time. In particular, we superimpose small random velocity fluctuations to the piston velocity and aim to obtain analytical solutions of the stochastic flow response. Within the context of small random fluctuations, we assume that the same thermodynamic conditions are valid as in the classical problem (i.e., that an isentropic region exists between the piston surface and the shock wave). This assumption is justified by the theory of weak perturbed shocks, although at the microscopic level, more complex processes may take place. Note also that the unperturbed shock can be strong. For example, it was reported in ref. 4 that a wall, which is adiabatic when rigidly fixed, may become conducting when it is allowed to have a stochastic motion independent of the value of its macroscopic velocity. However, in the macroscopic models that we develop here, we assume that such effects are negligible, and thus, all surfaces remain adiabatic.

In the first part of this article, we employ stochastic perturbation analysis to obtain closed-form analytical formulas for the perturbed shock paths. The random piston motion is modeled as a stochastic process following a Markov chain corresponding to various values of correlation length. The main physical finding extracted from the analytical solution is that the variance of the location of the perturbed shock grows quadratically with time at early times, but it switches to linear growth at later times. In the second part of this article, we perform high-resolution stochastic simulations by using a standard Monte Carlo approach and also the polynomial chaos method based on Wiener-Hermite expansions. The objective is to confirm the results of perturbation analysis and determine their validity range by using numerical solutions of the full nonlinear Euler equations subject to stochastic inputs. More generally, the stochastic piston problem that we have defined here serves as a strict testbed for rigorous evaluation of numerical stochastic solvers, and to this end, we have compared the performance of polynomial chaos against the Monte Carlo approach. The results depend critically on the specific value of correlation length, as well as on the length of time integration. At early times and/or large values of correlation length, the polynomial chaos method outperforms (often by orders of magnitude) the Monte Carlo approach. However, it is not as effective in other cases.

\section{Stochastic Perturbation Analysis}

We consider a piston having a constant velocity, $U_{p}$, moving into a straight tube filled with a homogeneous gas at rest. A shock wave will be generated ahead of the piston. A sketch of the piston-driven shock tube with superimposed random piston motion is shown in Fig. 1. Given the state ahead of the shock, the speed of the shock, $S$, and the thermodynamic states of the gas behind the shock (i.e., ahead of the piston) are determined in terms of the piston speed through the conservation of mass, momentum, and energy (5). For perfect gas with constant specific heats, these relations are as follows:

$$
\begin{aligned}
\frac{\rho_{0}}{\rho_{1}} & =1-\frac{U_{p}}{S}, \\
\frac{P_{1}}{P_{0}} & =1+\frac{\gamma}{c_{0}^{2}} S U_{p}, \text { and } \\
S & =\frac{\gamma+1}{4} U_{p}+\sqrt{\left(\frac{\gamma+1}{4} U_{p}\right)^{2}+C_{0}^{2}},
\end{aligned}
$$

where $\gamma=c_{p} / c_{v}$ is the ratio of specific heats, and $C_{0}$ is the local sound speed ahead of the shock. The sound speed behind the shock can be obtained as follows from Eqs. 1a and 1b:

$$
C^{2}=\left(C_{0}^{2}+\gamma S U_{p}\right)\left(1-U_{p} / S\right)
$$

In the following equations, we will normalize all velocities with $C_{0}$, and thus, $C_{0}=1$. We now define the stochastic motion of the piston by superimposing a small stochastic component to the steady speed of the piston, i.e.,

$$
u_{p}(t)=U_{p}[1+\varepsilon V(t, \omega)],
$$

where the amplitude $\varepsilon$ is small, $0<\varepsilon \ll 1$. Our objective is to find how the perturbed shock paths due to the random piston motion deviate from the unperturbed ones; these unperturbed paths are given as follows:

$$
X(t)=S \cdot t
$$

Under the small-amplitude assumption, the flow field induced by this perturbation can be obtained based on the assumption that the propagation speed in the region behind the shock and ahead of the piston can be identified as the propagation speed of the unperturbed flow quantities, i.e., $U_{p} \pm C$, where $C$ is the unperturbed sound speed behind the steadily moving shock as given by Eq. 2 .

This paper was submitted directly (Track II) to the PNAS office.

*To whom correspondence should be addressed. E-mail: gk@dam.brown.edu.

C 2004 by The National Academy of Sciences of the USA 


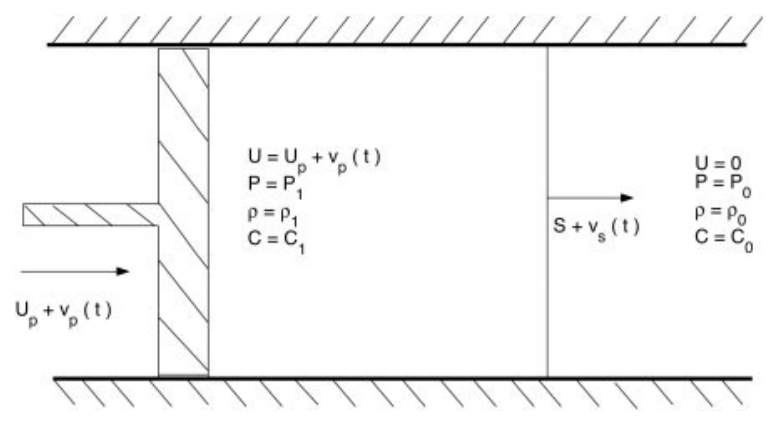

Fig. 1. Sketch of piston-driven shock tube with random piston motion.

To proceed, we consider the perturbed Riemann's invariants and evaluate them at the shock as follows:

$$
\begin{aligned}
(1 \pm k) v_{s}=j_{ \pm}= & v_{p} \pm \frac{2}{\gamma-1} a_{p}, \\
& \text { with } k=C \frac{S+S^{\prime} U_{p}}{1+\gamma S U_{p}},
\end{aligned}
$$

where $S^{\prime}=\left(d S / d U_{p}\right)$, and $v_{p}, a_{p}$, and $j_{ \pm}$are the perturbed piston velocity, the perturbed local sound speed, and the perturbed Riemann's invariants, respectively. These invariants are constant along the unperturbed (straight) characteristic lines. For more details on this derivation and the assumptions, see Supporting Text, which is published as supporting information on the PNAS web site. Fig. 2 shows a sketch of the shock paths induced by random piston motions. Specifically, the distorted lines show an instantaneous realization of the piston path and shock path. They are distorted because of induced reflections, as indicated in the plot by the characteristic lines. In the sketch, the steady and perturbed piston paths are indicated by $U_{p} t$ and $U_{p} t+\varepsilon \eta(t)$, whereas those for the shock paths are indicated by $S t$ and $S t+\varepsilon \xi(t)$. Also, $v_{p}\left(t_{2 n+1}\right)$ and $v_{s}\left(t_{2 n}\right)$ are on the forward characteristic $(d x / d t)=U_{p}+C ; v_{s}\left(t_{2 n+2}\right)$, whereas $v_{p}\left(t_{2 n+1}\right)$ is on the backward characteristic $(d x / d t)=U_{p}-$ $C$. Thus, we determine by the use of the Riemann invariants in Eq. 5, that:

$$
(1+k) v_{s}\left(t_{2 n}\right)=v_{p}\left(t_{2 n+1}\right)+\frac{2}{\gamma-1} a_{p}\left(t_{2 n+1}\right)
$$

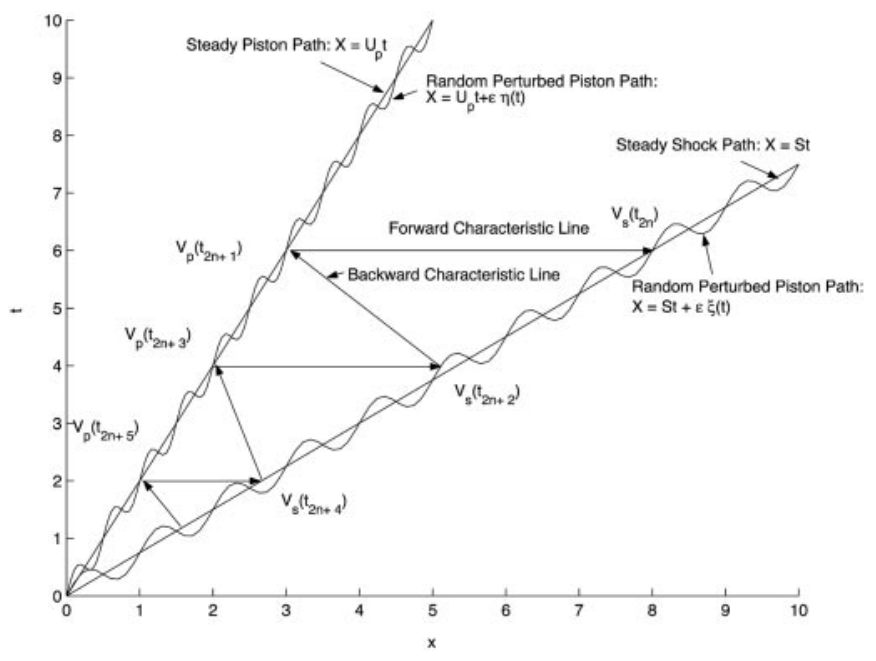

Fig. 2. Sketch of shock paths induced by random piston motions.

$$
(1-k) v_{s}\left(t_{2 n+2}\right)=v_{p}\left(t_{2 n+1}\right)-\frac{2}{\gamma-1} a_{p}\left(t_{2 n+1}\right) .
$$

By adding Eqs. $6 \mathbf{a}$ and $\mathbf{6 b}$ to eliminate $a_{p}\left(t_{2 n+1}\right)$, we obtain the following recurrence formula:

$$
v_{s}\left(t_{2 n}\right)=q v_{p}\left(t_{2 n+1}\right)-r v_{s}\left(t_{2 n+2}\right), \quad n=0, \ldots, N, \ldots,
$$

where

$$
q=\frac{2}{1+k} \quad \text { and } \quad r=\frac{1-k}{1+k}
$$

Eq. 7 defines a recursive relationship between the velocities at the shock and the perturbation of the piston motion $v_{p}(t)$. Starting at time $t_{0}$ and iterating up to $N$, we obtain from Eq. 7 a set of $(N+1)$ terms. By eliminating $v_{s}\left(t_{2}\right), v_{s}\left(t_{4}\right) \ldots v_{s}\left(t_{2 N}\right)$ from this set, we obtain the following:

$$
v_{s}(t)=q \sum_{n=0}^{N}(-r)^{n} v_{p}\left(t_{2 n+1}\right)+(-r)^{N+1} v_{s}\left(t_{2 N+2}\right) \text {. }
$$

If the perturbation of the piston starts at time $t_{s}>0$, the zigzag path of the characteristics coming down to the origin will end on the piston path; therefore, $v_{s}$ in the last term in Eq. 8 is zero. However, if $t_{s}=0$, the zigzag path will zigzag indefinitely to approach $t=0$, i.e., $N \rightarrow \infty$. Because $r$ is always less than unity, the last term of Eq. 8 will approach zero for any finite value of $v_{s}\left(t_{\infty}\right)$. Therefore, one can drop the last term in Eq. 8 to obtain the following:

$$
v_{s}(t)=q \sum_{n=0}^{N}(-r)^{n} v_{p}\left(t_{2 n+1}\right),
$$

where $N=\infty$ if $t_{s}=0$; i.e., the perturbation of the piston starts at $t=0$ or is determined by the last nonzero value of $v_{p}\left(t_{2 N+1}\right)$.

To make effective use of this relation, we need to obtain the relationship of the shock locations at $t_{2 n}, t_{2 n+1}$, and $t_{2 n+2}$. To this end, let us denote the shock locations at time $t_{2 n}$ and $t_{2 n+2}$ by $X_{s}\left(t_{2 n}\right)$ and $X_{s}\left(t_{2 n+2}\right)$, respectively, and the piston location at $t_{2 n+1}$ by $X_{p}\left(t_{2 n+1}\right)$. Assuming that the characteristics are approximated by straight lines with their slopes given by $U_{p} \pm C$, we have the following:

$$
\begin{aligned}
X_{s}\left(t_{2 n}\right)-X_{p}\left(t_{2 n+1}\right) & =\left(U_{p}+C\right)\left(t_{2 n}-t_{2 n+1}\right) \\
X_{p}\left(t_{2 n+1}\right)-X_{s}\left(t_{2 n+2}\right) & =\left(U_{p}-C\right)\left(t_{2 n+1}-t_{2 n+2}\right) .
\end{aligned}
$$

Also, by defining the perturbed path of the shock and the piston by $\xi(t)$ and $\eta(t)$, we can express the following:

$$
\begin{aligned}
X_{s}\left(t_{2 n}\right) & =S \cdot t_{2 n}+\varepsilon \xi\left(t_{2 n}\right), \\
X_{S}\left(t_{2 n+2}\right) & =S \cdot t_{2 n+2}+\varepsilon \xi\left(t_{2 n+2}\right), \text { and } \\
X_{p}\left(t_{2 n+1}\right) & =U_{p} t_{2 n+1}+\varepsilon \eta\left(t_{2 n+1}\right) .
\end{aligned}
$$

Finally, by substituting these into Eq. 10, we obtain the following:

$$
\begin{aligned}
& t_{2 n+1}=\alpha t_{2 n}+\frac{\varepsilon}{C}\left[\eta\left(t_{2 n+1}\right)-\xi\left(t_{2 n}\right)\right] \\
& t_{2 n+2}=\beta t_{2 n}+\varepsilon \gamma\left[2 \eta\left(t_{2 n+1}\right)-\xi\left(t_{2 n}\right)-\xi\left(t_{2 n+2}\right)\right],
\end{aligned}
$$

where

$$
\alpha=\frac{C+U_{p}-S}{C}<1,
$$




$$
\begin{aligned}
& \beta=\frac{C+U_{p}-S}{C+S-U_{p}}<1, \text { and } \\
& \gamma=\frac{1}{C+S-U_{p}}<1 .
\end{aligned}
$$

The above inequalities are due to $C>1$ and $S>U_{p}$.

If we drop the term containing $\varepsilon$ in Eq. 11, which is consistent with the small disturbance assumption, the recurrent relationship is very simplified and closed form solutions can be obtained. (We will retain this term in the subsection below.) With this simplification we have the following:

$$
t_{2 n+1}=\alpha t_{2 n}=\alpha \beta t_{2 n-2}=\alpha \beta^{2} t_{2 n-1} \cdots=\alpha \beta^{n} t_{0}=\alpha \beta^{n} t .
$$

Eq. 9 then reads as follows:

$$
v_{s}(t)=q \sum_{n=0}^{N}(-r)^{n} v_{p}\left(\alpha \beta^{n} t\right)
$$

and the shock speed is then obtained as follows:

$$
S\left(U_{p}+v_{s}(t)\right)=S\left(U_{p}\right)+\frac{d S}{d U_{p}} v_{s}(t)=S+S^{\prime} v_{s}(t),
$$

with the shock path governed by the following:

$$
\frac{d X_{s}}{d t}=S+S^{\prime} v_{s}(t)
$$

By using Eq. 12 and $X_{s}=S t+\varepsilon \xi(t)$ in the above equation, we have the following:

$$
\varepsilon \frac{d \xi}{d t}=q S^{\prime} \sum_{n=0}^{\infty}(-r)^{n} v_{p}\left(\alpha \beta^{n} t\right)
$$

and by taking $\xi(0)=0$, we obtain the following:

$$
\xi(t)=\frac{q S^{\prime}}{\varepsilon} \sum_{n=0}^{\infty}(-r)^{n} \int_{0}^{t} d t_{1} v_{p}\left(\alpha \beta^{n} t_{1}\right) .
$$

As a check of Eq. 12, we first consider the simple problem of a piston with its velocity subject to a small constant perturbation $v_{p}$ starting at $t=0$ (e.g., a step function of size $v_{p}$ ). According to Eq. 13, we have the following:

$$
v_{s}(t)=q \sum_{n=0}^{\infty}(-r)^{n} v_{p}\left(t_{2 n+1}\right)=q v_{p} \sum_{n=0}^{\infty}(-r)^{n}=v_{p} ;
$$

i.e., the velocity behind the shock will be $U_{p}+v_{p}$ for all $t>0$. The shock speed $S$ will change to $S\left(U_{p}+v_{p}\right)$, instead of $S\left(U_{p}\right)$.

We now consider $v_{p}$ to be a random process with zero mean and the following covariance:

$$
\left.\begin{array}{rl}
v_{p}(t) & =\varepsilon U_{p} V(t, \omega) \\
\langle V(t, \omega)\rangle & =0 \\
\left\langle V\left(t_{1}, \omega\right), V\left(t_{2}, \omega\right)\right\rangle & =e^{-\frac{\left|t_{1}-t_{2}\right|}{A}}
\end{array}\right\},
$$

where $A$ is the correlation time. The above covariance kernel describes a Markov random process in time. The larger the value of the correlation time $A$, the closer the random motion approaches a fully correlated process; we refer to this as a random variable case. However, the smaller the value of the correlation time $A$, the closer the motion resembles white noise.

By substituting Eq. 15a into 14, we obtain the following:

$$
\xi(t)=q S^{\prime} U_{p} \sum_{n=0}^{N}(-r)^{n} \int_{0}^{t} d t_{1} V\left(\alpha \beta^{n} t_{1}, \omega\right) .
$$

Because of Eq. 15b, we have the following:

$$
\langle\xi(t)\rangle=0
$$

$$
\begin{aligned}
\left\langle\xi^{2}(t)\right\rangle= & \left(U_{p} q S^{\prime}\right)^{2} \sum_{n=0}^{\infty} \sum_{m=0}^{\infty}(-r)^{m+n} \\
& \cdot \int_{0}^{t} d t_{1} \int_{0}^{t} d t_{2} e^{-\frac{\alpha}{A}\left|\beta^{n} t_{1}-\beta^{m} t_{2}\right|} .
\end{aligned}
$$

The double summation in Eq. $\mathbf{1 6}$ can be split into three parts, $(i)$ the sum of all diagonal terms, (ii) the sum of all the terms above the diagonal, and (iii) the terms described in detail below. It is evident that the last two sums are equal. Thus, we have the following:

$$
\begin{aligned}
\left\langle\xi^{2}(t)\right\rangle= & \left(U_{p} q S^{\prime}\right)^{2}\left[2 \sum_{n=1}^{\infty} \sum_{m=0}^{n-1}(-r)^{n+m} \int_{0}^{t} d t_{1}\right. \\
& \cdot \int_{0}^{t} d t_{2} e^{-\frac{\alpha}{A}\left|\beta^{n} t_{1}-\beta^{m} t_{2}\right|} \\
& \left.+\sum_{n=0}^{\infty}\left(r^{2 n}\right) \int_{0}^{t} d t_{1} \int_{0}^{t} d t_{2} e^{-\frac{\alpha \beta^{n}}{A}\left|t_{1}-t_{2}\right|}\right] .
\end{aligned}
$$

Both integrals in the above equation can be integrated explicitly to give the following:

$$
\begin{aligned}
\left\langle\xi^{2}(\tau)\right\rangle= & \left(U_{p} q S^{\prime} A / \alpha\right)^{2}\left[2 \sum_{n=1}^{\infty} \sum_{m=0}^{n-1}(-r)^{n+m} I_{n, m}(\tau)\right. \\
& \left.+\sum_{n=0}^{\infty} r^{2 n} I_{n, n}(\tau)\right]
\end{aligned}
$$

where $\tau=\alpha t / A$, and

$$
I_{n, m}(\tau)=\frac{2 \tau}{\beta^{m}}+\frac{1}{\beta^{n+m}}\left[e^{-\beta^{m} \tau}+e^{-\beta^{n} \tau}-1-e^{-\left(\beta^{m}-\beta^{n}\right) \tau}\right],
$$

where $m<n$. For $\tau \ll 1$, it is easy to show that $I_{n, m}=\tau^{2}$. The summations in Eq. 17 can be performed explicitly to obtain the following:

$$
\left\langle\xi^{2}(\tau)\right\rangle \approx\left(U_{p} q S^{\prime} A / \alpha\right)^{2} \frac{\tau^{2}}{(1+r)^{2}} \text { for } \tau \ll 1 .
$$

For $\tau \gg 1$, we can neglect the exponential terms in Eq. 18, and thus, 


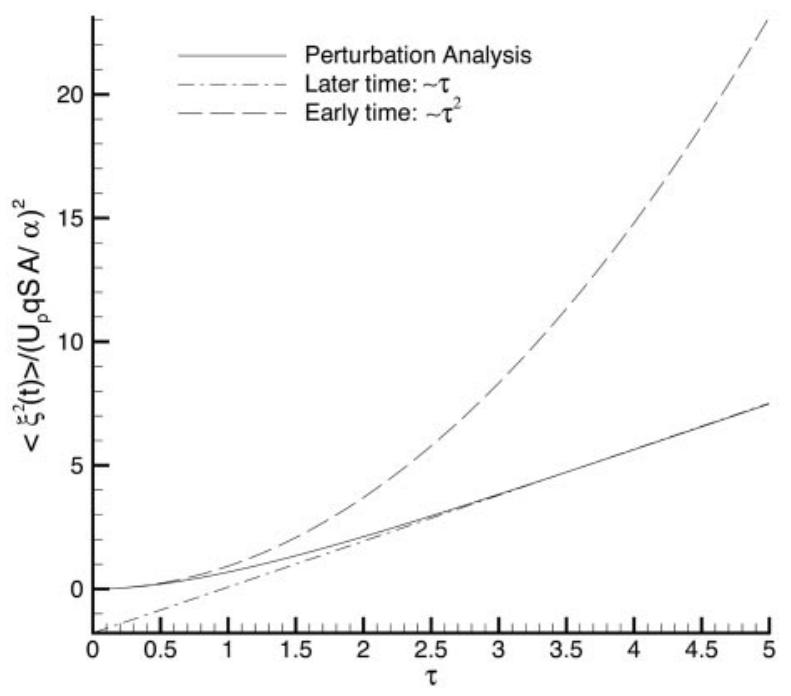

Fig. 3. Normalized variance of perturbed shock paths. The solid line indicates perturbation analysis results (see Eq. 17), the dashed line indicates early-time asymptotic results from Eq. 18, and the dashed-dotted line indicates late-time asymptotic results from Eq. 19.

$$
I_{n, m}(\tau)= \begin{cases}\frac{2 \tau}{\beta^{m}}-\frac{1}{\beta^{n+m}} & \text { for } m<n \\ \frac{2 \tau}{\beta^{n}}-\frac{2}{\beta^{2 n}} & \text { for } m=n .\end{cases}
$$

These expressions for $I_{n, m}$ can again be summed analytically on the right side of Eq. 17 to obtain the following:

$$
\begin{aligned}
\left\langle\xi^{2}(\tau)\right\rangle \approx & \left(U_{p} q S^{\prime} A / \alpha\right)^{2}\left[\frac{2 \tau(1-r)}{\left(1-r^{2} / \beta\right)(1+r)}\right. \\
& \left.-\frac{2}{\left(1-r^{2} / \beta^{2}\right)(1+r / \beta)}\right], \text { for } \tau \gg 1 .
\end{aligned}
$$

For arbitrary values of $\tau$, we calculate the quantities in square brackets in Eq. 17 numerically. Because of the smallness of the values of $r$ and $\beta$, the series converges quickly. Fig. 3 shows the quantity $\left\langle\xi^{2}(\tau)\right\rangle /\left(U_{p} q S^{\prime} A / \alpha\right)^{2}$ as a function of $\tau$ given by Eq. 17, with $U_{p}=1.25$, i.e., corresponding to Mach number of the shock $M=$ 2. The asymptotic formula for small and large $\tau$ given in Eqs. 18 and 19 are also included in the plot. We observe a qualitative change in the stochastic response versus time. At early times, the location of the path scales linearly with time, whereas at later times, it scales with square root of time [note that the variance $\langle\xi\rangle^{2} \sim(\text { length })^{2}$ ]. This interesting result is consistent with physical intuition, suggesting that at early times convective motions dominate, whereas at longer times, the diffusion process takes over.

Within the context of small-amplitude random motions of the piston, we have neglected the last term in Eq. 11 involving $\varepsilon$. This simplification allowed us to obtain closed-form analytical solutions, as we explained in the previous section. Now, we revisit this approximation and retain that term, so we employ the following recurrence formulas:

$$
\begin{aligned}
& t_{2 n+1}=\alpha t_{2 n}+\frac{\varepsilon}{C}\left[\eta\left(t_{2 n+1}\right)-\xi\left(t_{2 n}\right)\right], \text { and } \\
& t_{2 n+2}=\beta t_{2 n}+\varepsilon \gamma\left[2 \eta\left(t_{2 n+1}\right)-\xi\left(t_{2 n}\right)-\xi\left(t_{2 n+2}\right)\right],
\end{aligned}
$$

where $\alpha, \beta$, and $\gamma$ are given in Eq. 12. For a general $n$, we have the following sequence:

$$
\begin{aligned}
t_{2 n}= & \beta t_{2(n-1)}+\varepsilon \gamma\left[2 \eta\left(t_{2 n-1}\right)-\xi\left(t_{2(n-1)}\right)-\xi\left(t_{2 n}\right)\right] \\
t_{2(n-1)}= & \beta t_{2(n-2)}+\varepsilon \gamma\left[2 \eta\left(t_{2 n-3}\right)-\xi\left(t_{2(n-2)}\right)-\xi\left(t_{2(n-1)}\right)\right] \\
t_{2(n-2)}= & \beta t_{2(n-3)}+\varepsilon \gamma\left[2 \eta\left(t_{2 n-5}\right)-\xi\left(t_{2(n-3)}\right)-\xi\left(t_{2(n-2)}\right)\right] \\
& \cdots \\
t_{2}= & \beta t+\varepsilon \gamma\left[2 \eta\left(t_{1}\right)-\xi(t)-\xi\left(t_{2}\right)\right] .
\end{aligned}
$$

Solving for $t_{2 n}$ from the above equations, we obtain the following:

$$
\begin{aligned}
t_{2 n}= & \beta^{n} t+\varepsilon \gamma\left[\sum_{j=1}^{n} 2 \eta\left(t_{2 j-1}\right) \beta^{n-j}-(1+\beta)\right. \\
& \left.\cdot \sum_{j=1}^{n-1} \xi\left(t_{2 j}\right) \beta^{n-j-1}-\xi\left(t_{2 n}\right)-\beta^{n-1} \xi(t)\right] .
\end{aligned}
$$

Thus, $t_{2 n+1}$ can be expressed as follows:

$$
\begin{aligned}
t_{2 n+1}= & \beta^{n}\left[\alpha t-\frac{\varepsilon \xi(t)}{C}\right]+\frac{\varepsilon}{C}\left[2 \beta \sum_{j=1}^{n} \eta\left(t_{2 j-1}\right) \beta^{n-j}+\eta\left(t_{2 n+1}\right)\right] \\
& -\frac{\varepsilon(1+\beta)}{C} \sum_{j=1}^{n} \beta^{n-j} \xi\left(t_{2 j}\right) .
\end{aligned}
$$

The shock path is governed by the following:

$$
\varepsilon \frac{d \xi}{d t}=q S^{\prime} \sum_{n=0}^{\infty}(-r)^{n} v_{p}\left(t_{2 n+1}\right)
$$

and taking $\xi(0)=0$, we have the following:

$$
\xi(t)=\frac{q S^{\prime}}{\varepsilon} \sum_{n=0}^{\infty}(-r)^{n} \int_{0}^{t} d t_{1} v_{p}\left(t_{2 n+1}\right) .
$$

By considering Eqs. 15b and $\mathbf{2 3}$ together, we obtain the following:

$$
\begin{aligned}
\langle\xi(t)\rangle= & 0 \\
\left\langle\xi^{2}(t)\right\rangle= & \left(U_{p} q S^{\prime}\right)^{2} \sum_{n=0}^{\infty} \sum_{m=0}^{\infty}(-r)^{m+n} \int_{0}^{t} d t_{1} \\
& \cdot \int_{0}^{t} d t_{2} e^{-\frac{1}{A}\left|t_{1,2 n+1}-t_{2,2 m+1}\right|} .
\end{aligned}
$$

To compute the variance of the induced shock path $\left\langle\xi^{2}(t)\right\rangle$, we need to compute $t_{2 n+1}$. However, Eq. 22 shows that to compute $t_{2 n+1}$, we have to know the shock path $\xi(t)$ at all the previous reflection times $t_{2 j+1}$ and $t_{2 j}$. To this end, we solve Eqs. 21-24 numerically by employing an iteration method and setting $t_{2 j+1}=\alpha \beta i t$ and $t_{2 j}=\beta i t$ as an initial approximation. As the amplitude of the piston random motions becomes larger, the characteristic lines will no longer be linear. Eqs. 10 have to be modified.

In the following section, we perform stochastic numerical simulations to confirm our findings and establish limitations of the stochastic perturbation analysis presented in this section. 


\section{Stochastic Simulations}

We perform two types of stochastic simulations to verify the results of the previous section by following a Monte Carlo approach and a polynomial chaos approach. We employ the full nonlinear Euler equations with the extra complication that there is an unsteady stochastic boundary, namely, the piston position. To this end, a boundary-fitted coordinate approach is employed to transform the equations into a stationary domain. The transformed Euler equations contain stochastic source terms proportional to $-\rho \partial u_{p} / \partial t$ and $-\rho v \partial u_{p} / \partial t$ in the momentum and energy equations, respectively.

In Monte Carlo simulations, we use a Markov chain in time to represent the stochastic input. In polynomial chaos simulations, the representation of stochastic inputs is expressed by a KarhunenLoeve decomposition (see refs. 6 and 7). Specifically, we consider different representations of the stochastic inputs corresponding to the following covariance kernel:

$$
\left\langle V\left(t_{1}, \omega\right) V\left(t_{2}, \omega\right)\right\rangle=e^{\frac{-\left|t_{1}-t_{2}\right|}{A}},
$$

where $A$ is the correlation length. A corresponding Markov chain is employed to represent discretely the exponential kernel as follows:

$$
\begin{aligned}
V_{0}= & \xi_{0} \\
V_{1}= & C V_{0}+f \xi_{1} \\
& \cdots \\
V_{i+1}= & C V_{i}+f \xi_{i+1},
\end{aligned}
$$

where

$$
C=e^{\frac{-\Delta t}{A}} \text { and } f=\sqrt{1-C^{2}} .
$$

In the Monte Carlo simulation, a random piston velocity $u_{p}=$ $U_{p}\left(1+\varepsilon V_{i}(t, \omega)\right)$ is selected from the above Markov chain as a stochastic input at each time step $t_{i}$. In the polynomial chaos representation, we employ Wiener-Hermite expansions for all conservative and derived stochastic variables of the following form:

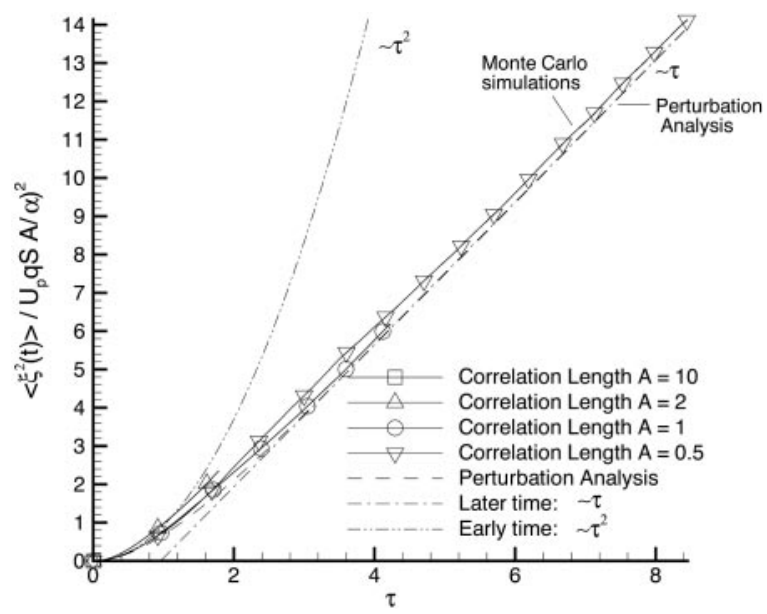

Fig. 4. Variance of the perturbed shock paths as a function of nondimensional time for different correlation lengths $(\varepsilon=0.01$, and $A=0.5,1,2$, and 10). The solid line corresponds to Monte Carlo simulations. The dashed line indicates results from perturbation analysis, the dash-dotted line indicates results from Eq. 19 for later time, and the dash-dot-dotted line indicates results from Eq. 18 for early time.

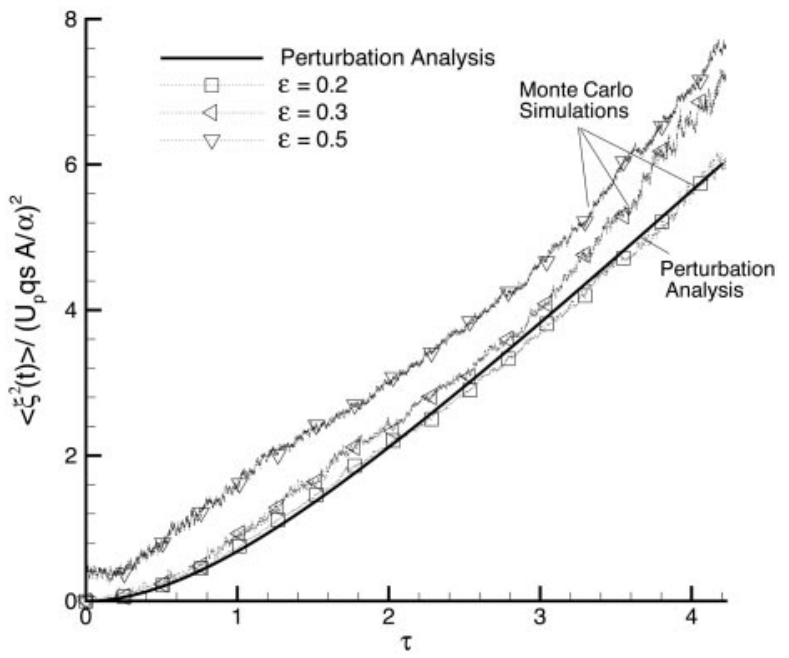

Fig. 5. Variance of the perturbed shock paths as a function of nondimensional time for different perturbation amplitudes $(A=1$, and $\varepsilon=0.2,0.3$, and $0.5)$. The dotted line corresponds to Monte Carlo simulations, and the solid line indicates results from perturbation analysis.

$$
X(\omega)=\sum_{j=0}^{M} \hat{x}_{j} \Phi_{j}(\xi(\omega)),
$$

where the basis $\left\{\Phi_{j}\right\}$ is formed from the Hermite orthogonal polynomials of degree $p$. Here, $\xi(\omega)$ is a Gaussian variable of dimension $N$ and $M$ is the total number of deterministic coefficients $\hat{x}_{j}$, where $M+1=(N+p) ! /(N ! p !)$. We employ the fifth-order weighted essentially nonoscillatory method in space in order to capture the shock location accurately and the third-order TVD Runge-Kutta method in time (see ref. 8 for details).

We now present some results for the following conditions. Behind the shock, we impose a steady piston velocity $U_{p}=1.25$ (normalized by the sound speed ahead of the shock), i.e., corresponding to Mach number of the shock $M=2$. Ahead of the shock, the sound speed is $C_{o}=1$ and the pressure is $P=1$. We investigate

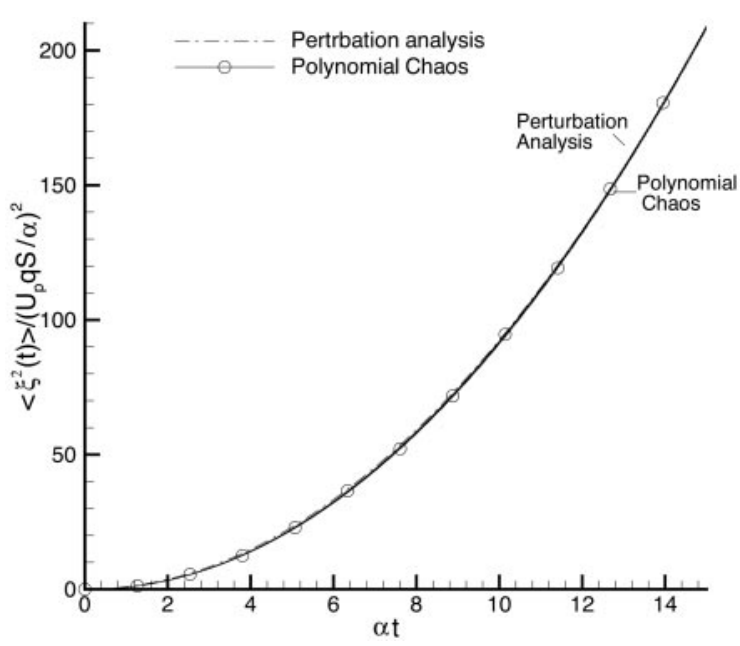

Fig. 6. Variance of the perturbed shock paths as a function of nondimensional time for a random variable (fully correlated kernel, $A \rightarrow \infty$ ) with amplitude $\varepsilon=0.01$. The dash-dotted line indicates results from perturbation analysis, and the solid line indicates numerical results from polynomial chaos simulations. 


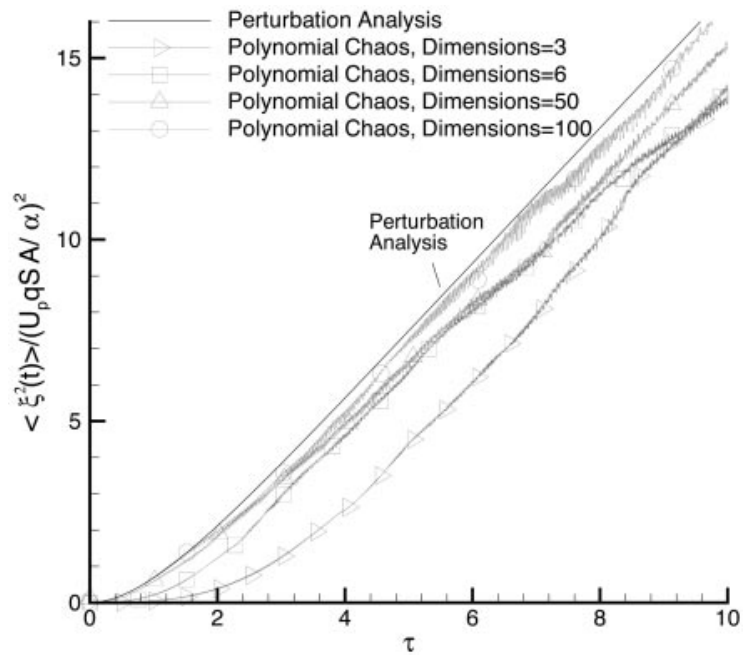

Fig. 7. Variance of the perturbed shock paths for $\varepsilon=0.01$ and $A=1$ obtained from polynomial chaos simulations with stochastic dimensions $N=$ $3,6,50$, and 100 .

the stochastic response for various values of the correlation length $A$ and of the amplitude of the random piston motion $\varepsilon$.

In Fig. 4, we plot the variance of the perturbed shock paths induced by small random piston motions corresponding to amplitude $\varepsilon=0.01$ and correlation lengths $A=0.5,1,2$, and 10, obtained from Monte Carlo simulations (2,000 runs). There is good agreement of the Monte Carlo solutions with the analytical solutions. In Fig. 5, we plot the variance of the perturbed shock paths induced by random piston motions corresponding to correlation length $A=$ 1 and amplitudes $\varepsilon=0.2,0.3$, and 0.5 obtained from Monte Carlo simulations (3,000 runs). For small amplitudes $\varepsilon=0.01,0.1$, and 0.2 , good agreement is observed between Monte Carlo simulations and analytical solution. However, for larger amplitudes, such as $\varepsilon=0.3$ and 0.5 , the stochastic simulation deviates from the analytical solution. We examine this discrepancy in more detail below, but first we present results from the polynomial chaos simulations.

Fig. 6 shows results from polynomial chaos simulations corresponding to piston motions described by a random variable, i.e., a fully correlated stochastic process by which $A \rightarrow \infty$. The polynomial chaos simulations match the exact analytical solutions quite closely (i.e., the variance of the shock location grows quadratically with time) even over a more than two-orders of magnitude change in the value of the variance. This result verifies the convergence of the Hermite chaos for this case. Fig. 7 shows results from polynomial chaos simulations corresponding to piston motions described by a random process with amplitude $\varepsilon=0.01$ and correlation time $A=$ 1. In the polynomial chaos simulations, the number of stochastic dimensions of random input is changed from $N=3,6,50$ to $N=$ 100 ( $N$ is also the number of Karhunen-Loeve modes for representing the stochastic piston motion). By increasing the dimensions of random input, the polynomial chaos simulations agree better with the analytical solution longer. However, there is a finite error after long-time integration, unlike the Monte Carlo simulations.

We now reexamine the effect of neglecting the second term in Eq. 11, which we included in the refined perturbation analysis of the

1. Courant, R. \& Friedrichs, K. O. (1948) Supersonic Flows and Shock Waves (Interscience, New York).

2. Whitham, G. B. (1974) Linear and Nonlinear Waves (Wiley, New York).

3. Lazarev, M. P., Prasad, P. \& Singh, S. K. (1995) ZAMP 46, 752-771.

4. Gruber, C. \& Frachebourg, L. (2004) On the Adiabatic Properties of a Stochastic Wall: Evolution, Stationary Non-Equilibrium, and Equilibrium States, preprint.

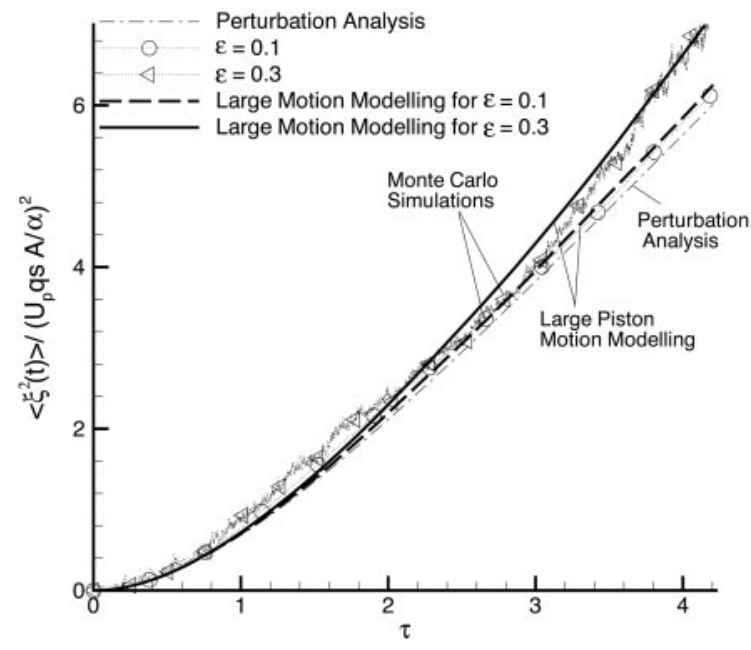

Fig. 8. Variance of the perturbed shock paths as a function of nondimensional time for relatively large piston motions $(A=1$, and $\varepsilon=0.1$ and 0.3 ). Thick solid and thick dashed lines correspond to large-amplitude perturbation analysis. The dotted line indicates results from Monte Carlo simulations, and the dashed-dotted line indicates results from small-amplitude perturbation analysis.

previous section. In Fig. 8, we compare the variance of the perturbed shock paths with large $U_{p}+v_{p}(t)$ random piston motions obtained from Monte Carlo simulations, analytical solutions from perturbation analysis, and analytical results obtained including the corrections for larger random piston motions. Significant improvement in the semianalytical results is evident compared with Monte Carlo simulations.

\section{Summary}

The stochastic piston problem is a reformulation, within the stochastic framework, of a classical aerodynamics problem that studies how small random piston motions affect shock paths. We have developed an analytical solution for the linearized Euler equations for the stochastic piston problem. Specifically, Eqs. 17-19 represent the main analytical results of this article. The first equation gives the full analytical expression, whereas the last two equations give asymptotic results for early and longer times, respectively. They reveal that the variance of the location of the perturbed shock paths initially grows quadratically with time and switches to linear dependence for longer times. The stochastic numerical simulations presented in this article confirm the results and show good agreement with the analytical solution for up to $20 \%$ amplitudes of the random piston motion compared with the mean steady motion.

This work was supported by the Computational Mathematics program of the Air Force Office of Scientific Research and the U.S. Department of Energy. Computations were performed at the Technology Center for Advanced Scientific Computing and Visualization at Brown University, the National Center for Supercomputing Applications at the University of Illinois at Urbana-Champaign, and the National Partnership for Advanced Computational Infrastructure at the University of California (San Diego).

5. Liepmann, H. W. \& Roshko, A. (1957) Elements of Gas Dynamics (Wiley, New York) 6. Ghanem, R. G. \& Spanos, P. (1991) Stochastic Finite Elemnets: A Spectral Approach (Springer, New York).

7. Xiu, D. \& Karniadakis, G. E. (2002) SIAM J. Sci. Comput. 24, 619644.

8. Jiang, G.-S. \& Shu, C.-W. (1996) J. Comput. Phys. 126, 202-228. 